\title{
Sentinel Lymph Node Mapping and Biopsy in Breast Cancer Patients During the COVID-19 Pandemic
}

\author{
Daniela Cocco, MD, and Stephanie A. Valente, DO \\ Breast Services, Cleveland Clinic Foundation, Cleveland, $\mathrm{OH}$
}

\section{LETTER TO THE EDITOR,}

The coronavirus disease 2019 (COVID-19) pandemic has caused significant clinical practice changes in the oncology field, with the goal of minimizing the risk of COVID infection while maintaining cancer treatment as a priority. Recently, the Annals of Surgical Oncology provided a comprehensive guideline of key measures to successfully navigate the challenges of cancer care during COVID. The guideline provides perspectives on creating a safe environment for surgical oncology care by redirecting the multidisciplinary model and surgery prioritization, while advocating for cancer patients. ${ }^{1}$

Several recommendations for the management of breast cancer patients have suggested, when clinically indicated, the implementation of preferential outpatient procedures, ${ }^{2}$ neoadjuvant endocrine therapy, ${ }^{3}$ and the omission or delay of radiation and/or reconstruction, ${ }^{4,5}$ with the goal of lowering the COVID exposure risks for patients and reducing the use of healthcare resources. However, guidelines on lymph node surgery for cancer staging are currently unavailable. The intent of this letter is to provide our experience on the management of the sentinel lymph node (SLN) biopsy procedure that aligns with the principles of the Annals of Surgical Oncology guidelines.

SLN biopsy continues to be the standard of care, with the use of a dual tracer technique (technetium-99 [99mTc] and blue dye) for the highest SLN detection sensitivity; however, the use of $99 \mathrm{mTc}$ requires an additional

(C) Society of Surgical Oncology 2021

First Received: 7 December 2020

Accepted: 10 December 2020;

Published Online: 11 January 2021

D. Cocco, MD

e-mail: danycocco@gmail.com preoperative visit to the Nuclear Medicine Department for radioisotope injection. This could potentially lead to unsafe patient and healthcare provider exposure, which could be avoided by replacing the $99 \mathrm{mT}$ mapping with a tracer that can be administered intraoperatively in addition to the blue dye.

Since 2016, SLN mapping using indocyanine green (ICG) dye and near infrared (NIR) fluorescence detection has been utilized at the Cleveland Clinic Breast Cancer Center. Our group published results demonstrating that the use of ICG for lymphatic mapping and SLN identification offers many potential advantages, including the ability to inject the material in the operating room while the patient is under anesthesia, and using NIR to visualize lymphatic anatomy and flow in real time, showing equivalent detection rates compared with $99 \mathrm{mTc}{ }^{6}$

The use of this alternative technique has been extremely useful for SLN mapping during the COVID pandemic. At the time of surgery, after induction of general anesthesia, the patient receives a subdermal injection of $2 \mathrm{~mL}$ of blue dye and an additional subdermal injection of $1 \mathrm{~mL}$ of $0.5 \%$ ICG solution into the subareolar region. The area is massaged toward the SLNs and the lymphatic drainage anatomy, and SLNs are then visualized in real time under fluorescence using an NIR camera. ${ }^{6}$ The ICG fluorescent lymph node(s) is/are identified and then confirmed as sentinel via the blue staining dual tracer visualized from the injected blue dye.

Despite the challenges caused by the COVID-19 pandemic, the ability to implement newer technologies is an invaluable armamentarium to maintain high-quality care. We believe the ICG-guided SLN mapping represents a safe and accurate alternative to the traditionally used $99 \mathrm{mTc}$ that allows limited risk of exposure for both patient and healthcare provider during the COVID-19 pandemic.

DISCLOSURES Daniela Cocco and Stephanie A. Valente declare no conflicts of interest. 


\section{REFERENCES}

1. Hwang ES, Balch CM, Balch GC, Feldman SM, Golshan M, Grobmyer SR, et al. Surgical oncologists and the COVID-19 pandemic: guiding cancer patients effectively through turbulence and change. Ann Surg Oncol. 2020;27(8):2600-13.

2. Zaniboni A, Ghidini M, Grossi F, Indini A, Trevisan F, Iaculli A, et al. A review of clinical practice guidelines and treatment recommendations for cancer care in the COVID-19 pandemic. Cancers (Basel). 2020;12(9):2452.

3. Martí C, Sánchez-Méndez JI. Neoadjuvant endocrine therapy for luminal breast cancer treatment: a first-choice alternative in times of crisis such as the COVID-19 pandemic. Ecancermedicalscience. 2020;14:1027.

4. Braunstein LZ, Gillespie EF, Hong L, Xu A, Bakhoum SF, Cuaron $\mathrm{J}$, et al. Breast radiation therapy under COVID-19 pandemic resource constraints-approaches to defer or shorten treatment from comprehensive cancer center in the United States. Adv Radiat Oncol. 2020;5(4):582-8.

5. Ganesh Kumar N, Kung TA. Guidelines for breast reconstruction during the COVID-19 pandemic: are we considering enough evidence? Breast J. 2020;26(10):2108-9.

6. Valente SA, Al-Hilli Z, Radford DM, Yanda C, Tu C, Grobmyer SR. Near infrared fluorescent lymph node mapping with indocyanine green in breast cancer patients: a prospective trial. J Am Coll Surg. 2019;228(4):672-8.

Publisher's Note Springer Nature remains neutral with regard to jurisdictional claims in published maps and institutional affiliations. 